\title{
Leukotriene Receptor Family
}

National Cancer Institute

\section{Source}

National Cancer Institute. Leukotriene Receptor Family. NCI Thesaurus. Code C118888.

A diverse group of transmembrane proteins that can initiate intracellular signaling

pathways when leukotrienes are bound to their extracellular, ligand binding domains. 